\title{
Spectral Properties of the Threshold Network Model
}

\author{
Yusuke Ide, Norio Konno, and Nobuaki Obata
}

Abstract. We study the spectral distribution of the threshold network model. The results contain an explicit description of the distribution and its asymptotic behavior.

\section{Introduction}

The threshold network model $\mathcal{G}_{n}(X, \theta)$, where $X$ is a random variable, $n \geq 2$ is an integer, and $\theta \in \mathbb{R}$ is a constant called a threshold, is a random graph on the vertex set $V=\{1,2, \ldots, n\}$ obtained as follows: Let $X_{1}, X_{2}, \ldots, X_{n}$ be independent copies of $X$ and draw an edge between two distinct vertices $i, j \in V$ if $X_{i}+X_{j}>\theta$. In other words, $\mathcal{G}_{n}(X, \theta)$ is specified by the random adjacency matrix $A=\left(A_{i j}\right)$ defined by

$$
A_{i j}= \begin{cases}I_{(\theta, \infty)}\left(X_{i}+X_{j}\right), & \text { if } i \neq j, \\ 0, & \text { otherwise }\end{cases}
$$

where $I_{B}$ denotes the indicator function of a set $B$.

As a small variant one may allow self-loops; see, for example, [Bose and Sen 07]. In this case the threshold network model is denoted by $\tilde{\mathcal{G}}_{n}(X, \theta)$, where two vertices $i, j \in V$ (possibly $i=j$ ) are connected if $X_{i}+X_{j}>\theta$. The adjacency matrix $\tilde{A}=\left(\tilde{A}_{i j}\right)$ is given by

$$
\tilde{A}_{i j}=I_{(\theta, \infty)}\left(X_{i}+X_{j}\right), \quad i, j \in V .
$$

The threshold network model has been extensively studied as a reasonable candidate model of real-world complex graphs (networks), which are often 
characterized by small diameters, high clustering, and power-law (scale-free) degree distributions [Albert and Barabási 02, Boccaletti et al. 06, Newman 03]. In fact, the threshold network model belongs to the so-called hidden variable models [Caldarelli et al. 02, Söderberg 02] and is known for being capable of generating scale-free networks. Their mean behavior [Boguñá and Pastor-Satorras 03, Caldarelli et al. 02, Fujihara et al. 09b, Hagberg et al. 06, Masuda et al. 04, Servedio et al. 04, Söderberg 02] and limit theorems [Fujihara et al. 09a, Ide et al. 07, Ide et al. 09, Konno et al. 05] for the degree, the clustering coefficients, the number of subgraphs, and the average distance have been analyzed. For related work, see also [Diaconis et al. 09, Ide et al. 07, Ide et al. 09, Konno et al. 05, Mahadev and Peled 95, Masuda et al. 05, Masuda and Konno 06].

Spectral properties of the threshold network model are also of interest. As a simple case, the binary threshold model appears in [Taraskin 05]. The strong law of large numbers and central limit theorem for the rank of the adjacency matrix of the model with self-loops are given by [Bose and Sen 07]. Eigenvalues and eigenvectors of the Laplacian matrix of the model have been studied in [Merris 94, Merris 98]. For general results of spectral analysis of graphs, see, for example, [Hora and Obata 07].

The main purpose of this paper is to study the spectral distribution, i.e., the distribution of the eigenvalues of the adjacency matrix of the threshold network model. Theorems 2.1 and 3.1 show the representations of the spectral distribution of the models. Moreover, we give some examples whose eigenvalues are asymptotically dominated by the special eigenvalues -1 and 0 . Theorem 4.3 covers the preceding study of the rank of the adjacency matrix [Bose and Sen 07].

This paper is organized as follows: In Section 2 we recall the hierarchical structure of the threshold network model and derive the spectral distribution of each sample graph (threshold graph). In Section 3 we obtain similar results for the threshold network model that admits self-loops. In Section 4 we derive some asymptotic behavior for the spectral distributions, and in Section 5 we give a simple example called the binary threshold model.

\section{Spectra of Threshold Graphs}

Each sample graph $G \in \mathcal{G}_{n}(X, \theta)$ has a hierarchical structure described by the so-called creation sequence, introduced in [Hagberg et al. 06]. Here we adopt a variant from [Diaconis et al. 09]. Each $G$ being determined by the values of random variables $X_{1}, X_{2}, \ldots, X_{n}$, we arrange them in increasing order: $X_{(1)} \leq$ $X_{(2)} \leq \cdots \leq X_{(n)}$. If $X_{(1)}+X_{(n)}>\theta$, we have

$$
\theta<X_{(1)}+X_{(n)} \leq X_{(2)}+X_{(n)} \leq \cdots \leq X_{(n-1)}+X_{(n)},
$$




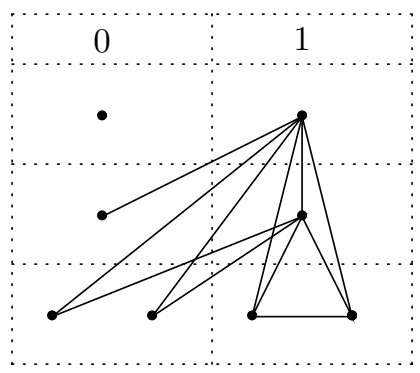

Figure I. A threshold graph $G$ corresponding to $S_{G}=\{1,1,0,0,1,0,1,0\}$.

which means that the vertex corresponding to $X_{(n)}$ is connected with the $n-1$ other vertices. Otherwise, we have

$$
\theta \geq X_{(1)}+X_{(n)} \geq \cdots \geq X_{(1)}+X_{(3)} \geq X_{(1)}+X_{(2)},
$$

which means that the vertex corresponding to $X_{(1)}$ is isolated. We set $s_{n}=1$ or $s_{n}=0$ according to whether the former case or the latter occurs. Then, according to the case we remove the random variable $X_{(n)}$ or $X_{(1)}$, and we use a similar procedure to define $s_{n-1}, \ldots, s_{2}$. Finally, we set $s_{1}=s_{2}$ and obtain a $\{0,1\}$-sequence $\left\{s_{1}, s_{2}, \ldots, s_{n}\right\}$, which is called the creation sequence of $G$ and is denoted by $S_{G}$.

Given a creation sequence $S_{G}$, let $k_{i}$ and $l_{i}$ denote the number of consecutive bits of 1's and 0's, respectively, as follows:

$$
S_{G}=\{\overbrace{1, \ldots, 1}^{k_{1}}, \overbrace{0, \ldots, 0}^{l_{1}}, \overbrace{1, \ldots, 1}^{k_{2}}, \overbrace{0, \ldots, 0}^{l_{2}}, \ldots, \overbrace{1, \ldots, 1}^{k_{m}}, \overbrace{0, \ldots, 0}^{l_{m}}\} .
$$

It may happen that $k_{1}=0$ or $l_{m}=0$, but we have $k_{2}, \ldots, k_{m}, l_{1}, \ldots, l_{m-1} \geq 1$, and $m \geq 1$. Moreover, by definition we have two cases: (a) $k_{1}=0$ (equivalently $s_{1}=0$ ) and $l_{1} \geq 2$; (b) $k_{1} \geq 2$ (equivalently $s_{1}=1$ ).

For example, if $S_{G}=\{1,1,0,0,1,0,1,0\}$, then $k_{1}=2, l_{1}=2, k_{2}=1, l_{2}=1$, $k_{3}=1, l_{3}=1$, and Figure 1 shows the shape of $G$.

The creation sequence $S_{G}$ gives rise to a partition of the vertex set:

$$
V=\bigcup_{i=1}^{m} V_{i}^{(1)} \cup \bigcup_{i=1}^{m} V_{i}^{(0)} \quad\left|V_{i}^{(1)}\right|=k_{i}, \quad\left|V_{i}^{(0)}\right|=l_{i} .
$$

The subgraph induced by $V_{i}^{(1)}$ is the complete graph on $k_{i}$ vertices, and that induced by $V_{i}^{(0)}$ is the null graph on $l_{i}$ vertices. Moreover, every vertex in $V_{i}^{(1)}$ (respectively $V_{i}^{(0)}$ ) is connected to (respectively disconnected from) all vertices in

$$
V_{1}^{(1)} \cup \cdots \cup V_{i}^{(1)} \cup V_{1}^{(0)} \cup \cdots \cup V_{i-1}^{(0)} .
$$


In general, a graph possessing the above hierarchical structure is called a threshold graph [Mahadev and Peled 95]. Hereinafter, we use $\delta_{\lambda}$ as the point measure on $\lambda \in \mathbb{R}$.

Theorem 2.I. Let $G$ be a threshold graph with a creation sequence $S_{G}=\left\{s_{1}=\right.$ $\left.s_{2}, s_{3}, \ldots, s_{n}\right\}$. Define $k_{i}$ and $l_{i}$ as in (2.1) and set

$$
C_{n}(-1)=\sum_{i=1}^{m} k_{i}-(m-1)-I_{\{1\}}\left(s_{1}\right), \quad C_{n}(0)=\sum_{i=1}^{m} l_{i}-(m-1) .
$$

Then the spectral distribution of $G$ is given by

$$
\mu_{n}(G)=\frac{C_{n}(-1)}{n} \delta_{-1}+\frac{C_{n}(0)}{n} \delta_{0}+\frac{1}{n} \sum_{j=1}^{J} \delta_{\lambda_{j}}, \quad J=2(m-1)+I_{\{1\}}\left(s_{1}\right),
$$

where $\left\{\lambda_{j}\right\}$ exhausts the eigenvalues of the matrix

$$
\left[\begin{array}{ccccccc}
k_{m}-1 & l_{m-1} & k_{m-1} & l_{m-2} & \ldots & l_{1} & k_{1} \\
k_{m} & 0 & 0 & 0 & \ldots & 0 & 0 \\
k_{m} & 0 & k_{m-1}-1 & l_{m-2} & \ldots & l_{1} & k_{1} \\
k_{m} & 0 & k_{m-1} & 0 & \ldots & 0 & 0 \\
\vdots & \vdots & \vdots & \vdots & \ddots & \vdots & \vdots \\
k_{m} & 0 & k_{m-1} & 0 & \ldots & 0 & 0 \\
k_{m} & 0 & k_{m-1} & 0 & \ldots & 0 & k_{1}-1
\end{array}\right]
$$

for $s_{1}=1$ (equivalently $k_{1} \geq 2$ ), or

$$
\left[\begin{array}{ccccccc}
k_{m}-1 & l_{m-1} & k_{m-1} & l_{m-2} & \ldots & k_{2} & l_{1} \\
k_{m} & 0 & 0 & 0 & \ldots & 0 & 0 \\
k_{m} & 0 & k_{m-1}-1 & l_{m-2} & \ldots & k_{2} & l_{1} \\
k_{m} & 0 & k_{m-1} & 0 & \ldots & 0 & 0 \\
\vdots & \vdots & \vdots & \vdots & \ddots & \vdots & \vdots \\
k_{m} & 0 & k_{m-1} & 0 & \ldots & k_{2}-1 & l_{1} \\
k_{m} & 0 & k_{m-1} & 0 & \ldots & k_{2} & 0
\end{array}\right]
$$

for $s_{1}=0$ (equivalently $k_{1}=0$ ). Moreover, any $\lambda_{j}$ in (2.3) differs from 0 and -1 , i.e., $C_{n}(-1)$ and $C_{n}(0)$ are respectively the multiplicities of the eigenvalues -1 and 0 . 
Proof. Let $\mathbf{1}_{i, j}$ denote the $i \times j$ matrix consisting of only 1 's, $\mathbf{0}_{i, j}$ the $i \times j$ zero matrix, $I_{i}$ the $i \times i$ identity matrix, and $\overline{\mathbf{1}}_{i, i}=\mathbf{1}_{i, i}-I_{i}$. By the hierarchical structure mentioned above, the adjacency matrix $A_{G}$ of $G$ is represented in the form

$$
\left[\begin{array}{cccccccc}
\mathbf{0}_{l_{m}, l_{m}} & \mathbf{0}_{l_{m}, k_{m}} & \mathbf{0}_{l_{m}, l_{m-1}} & \mathbf{0}_{l_{m}, k_{m-1}} & \mathbf{0}_{l_{m}, l_{m-2}} & \ldots & \mathbf{0}_{l_{m}, l_{1}} & \mathbf{0}_{l_{m}, k_{1}} \\
\mathbf{0}_{k_{m}, l_{m}} & \overline{\mathbf{1}}_{k_{m}, k_{m}} & \mathbf{1}_{k_{m}, l_{m-1}} & \mathbf{1}_{k_{m}, k_{m-1}} & \mathbf{1}_{l_{m}, l_{m-2}} & \ldots & \mathbf{1}_{k_{m}, l_{1}} & \mathbf{1}_{k_{m}, k_{1}} \\
\mathbf{0}_{l_{m-1}, l_{m}} & \mathbf{1}_{l_{m-1}, k_{m}} & \mathbf{0}_{l_{m-1}, l_{m-1}} & \mathbf{0}_{l_{m-1}, k_{m-1}} & \mathbf{0}_{l_{m-1}, l_{m-2}} & \ldots & \mathbf{0}_{l_{m-1}, l_{1}} & \mathbf{0}_{l_{m-1}, k_{1}} \\
\mathbf{0}_{k_{m-1}, l_{m}} & \mathbf{1}_{k_{m-1}, k_{m}} & \mathbf{0}_{k_{m-1}, l_{m-1}} & \overline{\mathbf{1}}_{k_{m-1}, k_{m-1}} & \mathbf{1}_{k_{m-1}, l_{m-2}} & \ldots & \mathbf{1}_{k_{m}-1}, l_{1} & \mathbf{1}_{k_{m}-1}, k_{1} \\
\mathbf{0}_{l_{m-2}, l_{m}} & \mathbf{1}_{l_{m-2}, l_{m}} & \mathbf{0}_{l_{m-2}, l_{m-1}} & \mathbf{1}_{l_{m-2}, k_{m-1}} & \mathbf{0}_{l_{m-2}, l_{m-2}} & \ldots & \mathbf{0}_{l_{m-2}, l_{1}} & \mathbf{0}_{l_{m-2}, k_{1}} \\
\vdots & \vdots & \vdots & \vdots & \vdots & \ddots & \vdots & \vdots \\
\mathbf{0}_{l_{1}, l_{m}} & \mathbf{1}_{l_{1}, k_{m}} & \mathbf{0}_{l_{1}, l_{m-1}} & \mathbf{1}_{l_{1}, k_{m-1}} & \mathbf{0}_{l_{1}, l_{m-2}} & \ldots & \mathbf{0}_{l_{1}, l_{1}} & \mathbf{0}_{l_{1}, k_{1}} \\
\mathbf{0}_{k_{1}, l_{m}} & \mathbf{1}_{k_{1}, k_{m}} & \mathbf{0}_{k_{1}, l_{m-1}} & \mathbf{1}_{k_{1}, k_{m-1}} & \mathbf{0}_{k_{1}, l_{m-2}} & \ldots & \mathbf{0}_{k_{1}, l_{1}} & \overline{\mathbf{1}}_{k_{1}, k_{1}}
\end{array}\right] .
$$

The adjacency matrix $A$ acts on $\mathbb{C}^{n}$ from the left. We define subspaces of $\mathbb{C}^{n}$ by

$$
\begin{aligned}
V_{i}(-1) & =\left\{\left[\begin{array}{c}
\mathbf{0}_{u_{i}+l_{i}} \\
\boldsymbol{\xi}_{k_{i}} \\
\mathbf{0}_{d_{i}}
\end{array}\right]: \xi_{1}+\xi_{2}+\cdots+\xi_{k_{i}}=0\right\}, \quad 1 \leq i \leq m, \\
V_{i}(0) & =\left\{\left[\begin{array}{c}
\mathbf{0}_{u_{i}} \\
\boldsymbol{\eta}_{l_{i}} \\
\mathbf{0}_{k_{i}+d_{i}}
\end{array}\right]: \eta_{1}+\eta_{2}+\cdots+\eta_{l_{i}}=0\right\}, \quad 1 \leq i \leq m-1, \\
V_{m}(0) & =\left\{\left[\begin{array}{c}
\boldsymbol{\eta}_{l_{m}} \\
\mathbf{0}_{k_{m}+d_{m}}
\end{array}\right]\right\},
\end{aligned}
$$

where

$$
\boldsymbol{\xi}_{k}=\left[\begin{array}{c}
\xi_{1} \\
\xi_{2} \\
\vdots \\
\xi_{k}
\end{array}\right], \quad \boldsymbol{\eta}_{l}=\left[\begin{array}{c}
\eta_{1} \\
\eta_{2} \\
\vdots \\
\eta_{l}
\end{array}\right], \quad \mathbf{1}_{j}=\left[\begin{array}{c}
1 \\
1 \\
\vdots \\
1
\end{array}\right], \quad \mathbf{0}_{j}=\left[\begin{array}{c}
0 \\
0 \\
\vdots \\
0
\end{array}\right]
$$

and

$$
u_{i}=\sum_{j=i+1}^{m}\left(l_{j}+k_{j}\right), \quad d_{i}=\sum_{j=1}^{i-1}\left(l_{j}+k_{j}\right) .
$$

Since $A_{G}$ acts on $V_{i}(-1)$ as the scalar operator with -1 , it possesses the eigenvalue -1 with multiplicity at least

$$
\sum_{i=1}^{m} \operatorname{dim} V_{i}(-1)=\sum_{i=1}^{m}\left(k_{i}-1\right)=\sum_{i=1}^{m} k_{i}-m
$$


if $k_{1} \geq 2$ (i.e., $s_{1}=1$ ), and

$$
\sum_{i=2}^{m} \operatorname{dim} V_{i}(-1)=\sum_{i=2}^{m}\left(k_{i}-1\right)=\sum_{i=2}^{m} k_{i}-(m-1)
$$

if $k_{1}=0$ (i.e., $s_{1}=0$ ). In any case, the multiplicity is at least $C_{n}(-1)$, defined in (2.2). Similarly, acting on $V_{i}(0)$ as a scalar operator with $0, A_{G}$ possesses the eigenvalues 0 with multiplicity at least $C_{n}(0)$.

Let $W$ be the orthogonal complement to $\bigoplus_{i=1}^{m}\left(V_{i}(-1) \oplus V_{i}(0)\right)$. The matrix representation of $A_{G}$ on $W$ with respect to the basis

$$
\boldsymbol{v}_{i}=\left[\begin{array}{c}
\mathbf{0}_{u_{i}+l_{i}} \\
\mathbf{1}_{k_{i}} \\
\mathbf{0}_{d_{i}}
\end{array}\right], \quad 1 \leq i \leq m
$$

and

$$
\boldsymbol{w}_{i}=\left[\begin{array}{c}
\mathbf{0}_{u_{i}} \\
\mathbf{1}_{l_{i}} \\
\mathbf{0}_{k_{i}+d_{i}}
\end{array}\right], \quad 1 \leq i \leq m-1
$$

is given by (2.4) or by (2.5) according as $k_{1} \geq 2$ or $k_{1}=0$. Then, one may verify easily that the eigenvalues of the matrices (2.4) and (2.5) are different from -1 and 0 .

Remark 2.2. After a simple calculation, we see that the eigenvalues $\lambda_{1}, \ldots, \lambda_{J}$ in (2.3) are obtained from the characteristic equations

$$
M(\lambda)=0
$$

where

$$
M(\lambda)=\operatorname{det}\left[\begin{array}{ccccccc}
k_{m}-1-\lambda & l_{m-1} & k_{m-1} & l_{m-2} & \ldots & l_{1} & k_{1} \\
k_{m} & -\lambda & 0 & 0 & \ldots & 0 & 0 \\
k_{m} & 0 & k_{m-1}-1-\lambda & l_{m-2} & \ldots & l_{1} & k_{1} \\
k_{m} & 0 & k_{m-1} & -\lambda & \ldots & 0 & 0 \\
\vdots & \vdots & \vdots & \vdots & \ddots & \vdots & \vdots \\
k_{m} & 0 & k_{m-1} & 0 & \ldots & -\lambda & 0 \\
k_{m} & 0 & k_{m-1} & 0 & \ldots & 0 & k_{1}-1-\lambda
\end{array}\right]
$$


if $k_{1} \geq 2$ (i.e., $s_{1}=1$ ) and

$$
M(\lambda)=\operatorname{det}\left[\begin{array}{ccccccc}
k_{m}-1-\lambda & l_{m-1} & k_{m-1} & l_{m-2} & \ldots & k_{2} & l_{1} \\
k_{m} & -\lambda & 0 & 0 & \ldots & 0 & 0 \\
k_{m} & 0 & k_{m-1}-1-\lambda & l_{m-2} & \ldots & k_{2} & l_{1} \\
k_{m} & 0 & k_{m-1} & -\lambda & \ldots & 0 & 0 \\
\vdots & \vdots & \vdots & \vdots & \ddots & \vdots & \vdots \\
k_{m} & 0 & k_{m-1} & 0 & \ldots & k_{2}-1-\lambda & l_{1} \\
k_{m} & 0 & k_{m-1} & 0 & \ldots & k_{2} & -\lambda
\end{array}\right]
$$

if $k_{1}=0$ (i.e., $s_{1}=0$ ). Simple calculation shows that

$$
M(-1)= \begin{cases}k_{1} \cdots k_{m} \cdot l_{1} \cdots l_{m-1}, & \text { if } k_{1} \geq 2\left(\text { i.e., } s_{1}=1\right), \\ k_{2} \cdots k_{m} \cdot\left(l_{1}-1\right) \cdot l_{2} \cdots l_{m-1}, & \text { otherwise }\end{cases}
$$

and

$$
M(0)= \begin{cases}\left(k_{1}-1\right) \cdot k_{2} \cdots k_{m} \cdot l_{1} \cdots l_{m-1}, & \text { if } k_{1} \geq 2\left(\text { i.e., } s_{1}=1\right), \\ k_{2} \cdots k_{m} \cdot l_{1} \cdots l_{m-1}, & \text { otherwise }\end{cases}
$$

from which we see also that the $\left\{\lambda_{j}\right\}$ contain neither -1 nor 0 .

\section{Spectra of Threshold Graphs with Self-Loops}

The idea of a creation sequence in Section 2 can be applied to the threshold network model that allows self-loops. With each $G \in \widetilde{\mathcal{G}}_{n}(X, \theta)$ we associate a creation sequence $\widetilde{S}_{G}=\left\{\tilde{s}_{1}, \tilde{s}_{2}, \ldots, \tilde{s}_{n}\right\}$ as follows: if $X_{(1)}+X_{(n)}>\theta$, we have

$$
\theta<X_{(1)}+X_{(n)} \leq X_{(2)}+X_{(n)} \leq \cdots \leq X_{(n-1)}+X_{(n)} \leq X_{(n)}+X_{(n)},
$$

which implies that the vertex corresponding to $X_{(n)}$ is connected with the $n-1$ other vertices and has a self-loop. Otherwise,

$$
\theta \geq X_{(1)}+X_{(n)} \geq \cdots \geq X_{(1)}+X_{(3)} \geq X_{(1)}+X_{(2)} \geq X_{(1)}+X_{(1)},
$$

which means that the vertex corresponding to $X_{(1)}$ is isolated and has no selfloops.

We set $\tilde{s}_{n}=1$ or $\tilde{s}_{n}=0$ depending on whether the former case or the latter occurs. Then, according to the case, we remove the random variable $X_{(n)}$ or $X_{(1)}$, and we employ a similar procedure to define $\tilde{s}_{n-1}, \ldots, \tilde{s}_{2}$. Finally, letting $X_{(*)}$ be the last remaining random variable, set $\tilde{s}_{1}=1$ if $X_{*}>\theta / 2$ and $\tilde{s}_{1}=0$ otherwise. In this case $G$ is called a threshold graph with self-loops associated 
with a creation sequence $\tilde{S}=\left\{\tilde{s}_{1}, \tilde{s}_{2}, \ldots, \tilde{s}_{n}\right\}$. We note that if $\tilde{s}_{j}=1$, the corresponding vertex has a self-loop, and otherwise no self-loop.

Given a creation sequence $\tilde{S}=\left\{\tilde{s}_{1}, \tilde{s}_{2}, \ldots, \tilde{s}_{n}\right\}$, we define $k_{j}$ and $l_{j}$ as in (2.1). It may happen that $k_{1}=0$ and $l_{m}=0$, but $k_{2}, \ldots, k_{m}, l_{1}, \ldots, l_{m-1} \geq 1$ and $m \geq 1$. The adjacency matrix $\tilde{A}_{G}$ of $G$ is of the form

$$
\left[\begin{array}{cccccccc}
\mathbf{0}_{l_{m}, l_{m}} & \mathbf{0}_{l_{m}, k_{m}} & \mathbf{0}_{l_{m}, l_{m-1}} & \mathbf{0}_{l_{m}, k_{m-1}} & \mathbf{0}_{l_{m}, l_{m-2}} & \ldots & \mathbf{0}_{l_{m}, l_{1}} & \mathbf{0}_{l_{m}, k_{1}} \\
\mathbf{0}_{k_{m}, l_{m}} & \mathbf{1}_{k_{m}, k_{m}} & \mathbf{1}_{k_{m}, l_{m-1}} & \mathbf{1}_{k_{m}, k_{m-1}} & \mathbf{1}_{l_{m}, l_{m-2}} & \ldots & \mathbf{1}_{k_{m}, l_{1}} & \mathbf{1}_{k_{m}, k_{1}} \\
\mathbf{0}_{l_{m-1}, l_{m}} & \mathbf{1}_{l_{m-1}, k_{m}} & \mathbf{0}_{l_{m-1}, l_{m-1}} & \mathbf{0}_{l_{m-1}, k_{m-1}} & \mathbf{0}_{l_{m-1}, l_{m-2}} & \ldots & \mathbf{0}_{l_{m-1}, l_{1}} & \mathbf{0}_{l_{m-1}, k_{1}} \\
\mathbf{0}_{k_{m-1}, l_{m}} & \mathbf{1}_{k_{m-1}, k_{m}} & \mathbf{0}_{k_{m-1}, l_{m-1}} & \mathbf{1}_{k_{m}-1}, k_{m}-1 & \mathbf{1}_{k_{m-1}, l_{m}-2} & \ldots & \mathbf{1}_{k_{m}-1}, l_{1} & \mathbf{1}_{k_{m-1}}, k_{1} \\
\mathbf{0}_{l_{m-2}, l_{m}} & \mathbf{1}_{l_{m-2}, l_{m}} & \mathbf{0}_{l_{m-2}, l_{m-1}} & \mathbf{1}_{l_{m-2}, k_{m-1}} & \mathbf{0}_{l_{m-2}, l_{m-2}} & \ldots & \mathbf{0}_{l_{m-2}, l_{1}} & \mathbf{0}_{l_{m-2}, k_{1}} \\
\vdots & \vdots & \vdots & \vdots & \vdots & \ddots & \vdots & \vdots \\
\mathbf{0}_{l_{1}, l_{m}} & \mathbf{1}_{l_{1}, k_{m}} & \mathbf{0}_{l_{1}, l_{m-1}} & \mathbf{1}_{l_{1}, k_{m-1}} & \mathbf{0}_{l_{1}, l_{m-2}} & \ldots & \mathbf{0}_{l_{1}, l_{1}} & \mathbf{0}_{l_{1}, k_{1}} \\
\mathbf{0}_{k_{1}, l_{m}} & \mathbf{1}_{k_{1}, k_{m}} & \mathbf{0}_{k_{1}, l_{m-1}} & \mathbf{1}_{k_{1}, k_{m-1}} & \mathbf{0}_{k_{1}, l_{m-2}} & \ldots & \mathbf{0}_{k_{1}, l_{1}} & \mathbf{1}_{k_{1}, k_{1}}
\end{array}\right] .
$$

Repeating a similar argument as in Theorem 2.1, we obtain the following result.

Theorem 3.I. Let $G$ be a threshold graph with self-loops associated with a creation sequence $\tilde{S}=\left\{\tilde{s}_{1}, \tilde{s}_{2}, \ldots, \tilde{s}_{n}\right\}$ and its adjacency matrix given as in (3.1). Set

$$
\widetilde{C}_{n}(0)=n-2(m-1)-I_{\{1\}}\left(\tilde{s}_{1}\right) .
$$

Then the spectral distribution of $G$ is given by

$$
\widetilde{\mu}_{n}(G)=\frac{\widetilde{C}_{n}(0)}{n} \delta_{0}+\frac{1}{n} \sum_{j=1}^{J} \delta_{\lambda_{j}}, \quad J=2(m-1)+I_{\{1\}}\left(\tilde{s}_{1}\right),
$$

where $\left\{\lambda_{j}\right\}$ exhaust the eigenvalues of

$$
\left[\begin{array}{ccccccc}
k_{m} & l_{m-1} & k_{m-1} & l_{m-2} & \ldots & l_{1} & k_{1} \\
k_{m} & 0 & 0 & 0 & \ldots & 0 & 0 \\
k_{m} & 0 & k_{m-1} & l_{m-2} & \ldots & l_{1} & k_{1} \\
k_{m} & 0 & k_{m-1} & 0 & \ldots & 0 & 0 \\
\vdots & \vdots & \vdots & \vdots & \ddots & \vdots & \vdots \\
k_{m} & 0 & k_{m-1} & 0 & \ldots & 0 & 0 \\
k_{m} & 0 & k_{m-1} & 0 & \ldots & 0 & k_{1}
\end{array}\right]
$$

for $\tilde{s}_{1}=1$ (i.e., $k_{1} \geq 1$ ), or

$$
\left[\begin{array}{ccccccc}
k_{m} & l_{m-1} & k_{m-1} & l_{m-2} & \ldots & k_{2} & l_{1} \\
k_{m} & 0 & 0 & 0 & \ldots & 0 & 0 \\
k_{m} & 0 & k_{m-1} & l_{m-2} & \ldots & k_{2} & l_{1} \\
k_{m} & 0 & k_{m-1} & 0 & \ldots & 0 & 0 \\
\vdots & \vdots & \vdots & \vdots & \ddots & \vdots & \vdots \\
k_{m} & 0 & k_{m-1} & 0 & \ldots & k_{2} & l_{1} \\
k_{m} & 0 & k_{m-1} & 0 & \ldots & k_{2} & 0
\end{array}\right]
$$


for $\tilde{s}_{1}=0$ (i.e., $k_{1}=0$ ). Moreover, any $\lambda_{j}$ in (3.3) differs from 0 , i.e., $\widetilde{C}_{n}(0)$ is the multiplicity of 0 .

Remark 3.2. The eigenvalues $\lambda_{1}, \ldots, \lambda_{J}$ in (3.3) are obtained from the characteristic equations

$$
\operatorname{det}\left[\begin{array}{ccccccccc}
-\lambda & 0 & \ldots & 0 & 0 & 0 & \ldots & 0 & k_{m} \\
\lambda & -\lambda & \ldots & 0 & 0 & 0 & \ldots & k_{m-1} & 0 \\
\vdots & \ddots & \ddots & \vdots & \vdots & \vdots & \ldots & \vdots & \vdots \\
0 & 0 & \ddots & -\lambda & 0 & k_{2} & \ldots & 0 & 0 \\
0 & 0 & \ldots & \lambda & k_{1}-\lambda & 0 & \ldots & 0 & 0 \\
0 & 0 & \ldots & l_{1} & \lambda & -\lambda & \ldots & 0 & 0 \\
\vdots & \vdots & \vdots & \vdots & \vdots & \ddots & \ddots & \vdots & \vdots \\
0 & l_{m-2} & \ldots & 0 & 0 & 0 & \ddots & -\lambda & 0 \\
l_{m-1} & 0 & \ldots & 0 & 0 & 0 & \ldots & \lambda & -\lambda
\end{array}\right]=0
$$

for $s_{1}=1$ (i.e., $k_{1} \geq 1$ ), and

$$
\operatorname{det}\left[\begin{array}{cccccccccc}
-\lambda & 0 & \ldots & 0 & 0 & 0 & 0 & \ldots & 0 & k_{m} \\
\lambda & -\lambda & \ldots & 0 & 0 & 0 & 0 & \ldots & k_{m-1} & 0 \\
0 & \lambda & \ddots & 0 & 0 & 0 & 0 & \ldots & 0 & 0 \\
\vdots & \vdots & \ddots & \ddots & \vdots & \vdots & \vdots & \ldots & \vdots & \vdots \\
0 & 0 & \ldots & \lambda & -\lambda & k_{2} & 0 & \ldots & 0 & 0 \\
0 & 0 & \ldots & 0 & l_{1}+\lambda & -\lambda & 0 & \ldots & 0 & 0 \\
0 & 0 & \ldots & l_{2} & 0 & \lambda & -\lambda & \ldots & 0 & 0 \\
\vdots & \vdots & \vdots & \vdots & \vdots & \vdots & \ddots & \ddots & \vdots & \vdots \\
0 & l_{m-2} & \ldots & 0 & 0 & 0 & 0 & \ddots & -\lambda & 0 \\
l_{m-1} & 0 & \ldots & 0 & 0 & 0 & 0 & \ldots & \lambda & -\lambda
\end{array}\right]=0
$$

for $s_{1}=0$ (i.e., $\left.k_{1}=0\right)$.

\section{Limit Theorems}

In this section we discuss the asymptotic behavior of the spectral distributions obtained in the previous sections.

We first consider the case in which the distribution of $X$ is discrete and given by

$$
\mathbb{P}(X=i)=p_{i}, \quad i=0,1, \ldots, \quad \sum_{i=0}^{\infty} p_{i}=1
$$


Let $m \geq 1$ be a fixed integer. Take a particular threshold $\theta=2 m-1$ and assume that $p_{i}>0$ for $i=0,1, \ldots, 2 m-1$. It follows from the strong law of large numbers that

$$
\begin{aligned}
l_{i} & =\sharp\left\{j: X_{j}=m-i\right\}, \quad i=1, \ldots, m, \\
k_{i} & =\sharp\left\{j: X_{j}=m-1+i\right\}, \quad i=1, \ldots, m-1, \\
k_{m} & =\sharp\left\{j: X_{j} \geq 2 m-1\right\}, \\
l_{i} & =k_{i}=0, \quad i \geq m+1,
\end{aligned}
$$

for large $n$ almost surely. Moreover, denoting by $F$ the distribution function of $X$, we have

$$
\lim _{n \rightarrow \infty} \frac{1}{n} \sum_{i=1}^{m} l_{i}=F(m-1) \text { a.s. } \quad \lim _{n \rightarrow \infty} \frac{1}{n} \sum_{i=1}^{m} k_{i}=1-F(m-1) \text { a.s. }
$$

With these observation we easily obtain the following theorem.

Theorem 4.I. With notation and assumptions as above, the spectral distributions of $\mathcal{G}_{n}(X, 2 m-1)$ satisfy

$$
\lim _{n \rightarrow \infty} \mu_{n}(G)=(1-F(m-1)) \cdot \delta_{-1}+F(m-1) \cdot \delta_{0} \text { a.s. }
$$

Similarly, the spectral distributions of $\tilde{\mathcal{G}}_{n}(X, 2 m-1)$ satisfy

$$
\lim _{n \rightarrow \infty} \widetilde{\mu}_{n}(G)=\delta_{0} \text { a.s. }
$$

Remark 4.2. Similar results hold when the distribution of $X$ is discrete and $F$ has only a finite number of jumps in $(-\infty, \theta / 2]$ or $(\theta / 2, \infty)$. But no simple description is known for the general case.

Next we consider the case in which the distribution of $X$ is continuous. As is stated implicitly in [Bose and Sen 07], if the distribution of $X$ is continuous and symmetric around 0 , then the distribution of zero and one entries in the creation sequence $\widetilde{S}$ of each graph generated by $\widetilde{\mathcal{G}}_{n}(X, 0)$ is the same as the distribution of a sequence of i.i.d. Bernoulli random variables $\left\{\widetilde{Y}_{i}\right\}_{i=1,2, \ldots, n}$ with success probability $1 / 2$, that is,

$$
\mathbb{P}\left(\widetilde{Y}_{i}=0\right)=\mathbb{P}\left(\widetilde{Y}_{i}=1\right)=1 / 2, \quad \text { for } i=1,2, \ldots, n .
$$

In this case, we can take $\widetilde{Y}_{i}=I_{[0, \infty)}\left(X_{i}\right)$ by the first argument of the proof of [Bose and Sen 07, Theorem 1]. 
This means that $\widetilde{S}=\left\{\tilde{s}_{1}, \tilde{s}_{2}, \ldots, \tilde{s}_{n}\right\} \stackrel{d}{=}\left\{\widetilde{Y}_{1}, \widetilde{Y}_{2}, \ldots, \widetilde{Y}_{n}\right\}$. Recall that the creation sequence $S$ of each graph generated by $\mathcal{G}_{n}(X, 0)$ is always satisfied with $s_{1}=s_{2}$. Then we observe that $S=\left\{s_{1}=s_{2}, s_{3}, \ldots, s_{n}\right\} \stackrel{d}{=}\left\{Y_{2}, Y_{2}, Y_{3}, \ldots, Y_{n}\right\}$, where similarly, $\left\{Y_{i}\right\}_{i=2,3, \ldots, n}$ is the sequence of i.i.d. Bernoulli random variables with success probability $1 / 2$.

Taking the above consideration into account, we obtain the asymptotic behavior of coefficients of point measures on -1 and 0 appearing in $\mu_{n}(G)$ and $\widetilde{\mu}_{n}(G)$.

Theorem 4.3. Assume that the distribution of $X$ is continuous and symmetric around 0. Define $C_{n}(-1), C_{n}(0)$, and $\widetilde{C}_{n}(0)$ as in (2.2) and (3.2). Then we have

(1) $\lim _{n \rightarrow \infty} C_{n}(-1) / n=\lim _{n \rightarrow \infty} C_{n}(0) / n=1 / 4$ a.s.

(2) $\sqrt{n}\left(C_{n}(-1) / n-1 / 4\right) \Rightarrow N(0,1 / 4)$ and $\sqrt{n}\left(C_{n}(0) / n-1 / 4\right) \Rightarrow N(0,1 / 4)$ as $n \rightarrow \infty$.

(3) $\lim _{n \rightarrow \infty} \widetilde{C}_{n}(0) / n=1 / 2$ a.s.

(4) $\sqrt{n}\left(\widetilde{C}_{n}(0) / n-1 / 2\right) \Rightarrow N(0,1 / 4)$ as $n \rightarrow \infty$.

Proof. Note the following relations:

$$
\begin{aligned}
C_{n}(-1) & =\sum_{i=1}^{m} k_{i}-(m-1)-I_{\{1\}}\left(s_{1}\right) \\
& \stackrel{d}{=}\left(Y_{2}+\sum_{i=2}^{n} Y_{i}\right)-\sum_{i=2}^{n-1}\left(1-Y_{i}\right) Y_{i+1}-Y_{2}=Y_{2}+\sum_{i=2}^{n-1} Y_{i} Y_{i+1},
\end{aligned}
$$

$C_{n}(0)=\sum_{i=1}^{m} l_{i}-(m-1)$

$$
\begin{aligned}
& \stackrel{d}{=}\left\{\left(1-Y_{2}\right)+\sum_{i=2}^{n}\left(1-Y_{i}\right)\right\}-\sum_{i=2}^{n-1}\left(1-Y_{i}\right) Y_{i+1} \\
& =2-Y_{2}-Y_{n}+\sum_{i=2}^{n-1}\left(1-Y_{i}\right)\left(1-Y_{i+1}\right),
\end{aligned}
$$

$\widetilde{C}_{n}(0)=n-2(m-1)-I_{\{1\}}\left(\tilde{s}_{1}\right)$

$$
\stackrel{d}{=} n-2 \sum_{i=1}^{n-1}\left(1-\tilde{Y}_{i}\right) \widetilde{Y}_{i+1}-\widetilde{Y}_{1}=1-\widetilde{Y}_{n}+\sum_{i=1}^{n-1}\left(1-\tilde{Y}_{i}+\widetilde{Y}_{i+1}\right)\left(1+\widetilde{Y}_{i}-\widetilde{Y}_{i+1}\right) .
$$


We then easily check that

$$
\mathbb{E}\left[C_{n}(-1)\right]=\mathbb{E}\left[C_{n}(0)\right]-\frac{1}{2}=\frac{n}{4}
$$

and

$$
\mathbb{E}\left[\widetilde{C}_{n}(0)\right]=\frac{n}{2}
$$

Applying a similar argument as in [Bose and Sen 07, Theorem 1], we have the assertion of the theorem.

When the distribution of $X$ is continuous and symmetric around $\theta / 2$, we can obtain similar results for $\mathcal{G}_{n}(X, \theta)$ and $\widetilde{\mathcal{G}}_{n}(X, \theta)$ by a straightforward modification. Research covering a more general situation is now in progress.

\section{Binary Threshold Model}

In this section we give a simple example. The threshold network model defined by Bernoulli trials $X_{1}, X_{2}, \ldots, X_{n}$ with success probability $p$, i.e., $0<P\left(X_{i}=\right.$ $1)=p<1$, and a threshold $0 \leq \theta<1$ is called the binary threshold model and is denoted by $\mathcal{G}_{n}(p)$. For $G \in \mathcal{G}_{n}(p)$ the partition of the vertex set $V$ is given by

$$
V=V^{(1)} \cup V^{(0)}, \quad V^{(1)}=\left\{i ; X_{i}=1\right\}, \quad V^{(0)}=\left\{i ; X_{i}=0\right\} .
$$

Theorem 5.I. For $G \in \mathcal{G}_{n}(p)$ we set $\left|V^{(1)}\right|=k$ and $\left|V^{(0)}\right|=l$. Then the spectral distribution of $G$ is given by

$$
\mu_{k, l}=\frac{k-1}{n} \delta_{-1}+\frac{l-1}{n} \delta_{0}+\frac{1}{n} \delta_{\lambda_{+}}+\frac{1}{n} \delta_{\lambda_{-}},
$$

where

$$
\lambda_{ \pm}=\frac{k-1 \pm \sqrt{(k-1)^{2}+4 k l}}{2}
$$

Proof. We have only to apply Theorem 2.1 with $l_{1}=l, l_{2}=k_{1}=0, k_{2}=k$, and $m=2$. In this case, (2.5) becomes

$$
\left[\begin{array}{cc}
k-1 & l \\
k & 0
\end{array}\right],
$$

the eigenvalues of which are $\lambda_{ \pm}$in (5.1). 
Corollary 5.2. Let $\mu_{n}(G)$ be the spectral distribution of $G \in \mathcal{G}_{n}(p)$. Then we have

$$
\lim _{n \rightarrow \infty} \mu_{n}(G)=p \cdot \delta_{-1}+(1-p) \cdot \delta_{0} \text { a.s. }
$$

Proof. The result follows from the strong law of large numbers; see also Theorem 4.1.

As for the the mean spectral distribution, we have the following theorem.

Theorem 5.3. The mean spectral distribution of the binary threshold model $\mathcal{G}_{n}(p)$ is given by

$$
\begin{aligned}
\mu_{n}= & \left(p-\frac{1}{n}\right) \delta_{-1}+\left(1-p-\frac{1}{n}\right) \delta_{0} \\
& +\frac{1}{n} \sum_{k=0}^{n}\left(\begin{array}{l}
n \\
k
\end{array}\right) p^{k}(1-p)^{n-k}\left(\delta_{\lambda_{-}(k)}+\delta_{\lambda_{+}(k)}\right),
\end{aligned}
$$

where

$$
\lambda_{ \pm}(k)=\frac{k-1 \pm \sqrt{(k-1)^{2}+4 k(n-k)}}{2}, \quad k=0,1, \ldots, n
$$

Proof. Since

$$
P\left(\left|V^{(1)}\right|=k,\left|V^{(0)}\right|=l\right)=\left(\begin{array}{l}
n \\
k
\end{array}\right) p^{k}(1-p)^{l}, \quad k+l=n,
$$

the mean spectral distribution is given by

$$
\mu=\sum_{k=0}^{n}\left(\begin{array}{l}
n \\
k
\end{array}\right) p^{k}(1-p)^{l} \mu_{k, l} .
$$

Then (5.2) follows from Theorem 5.1 by direct computation.

Corollary 5.4. Let $\mu_{n}$ be the mean spectral distribution of the binary threshold model $\mathcal{G}_{n}(p)$. Then we have

$$
\lim _{n \rightarrow \infty} \mu_{n}=p \cdot \delta_{-1}+(1-p) \cdot \delta_{0}
$$

Acknowledgments. The second author has been funded by the Grant-in-Aid for Scientific Research (C) of the Japan Society for the Promotion of Science (Grant No. 21540118). The third author has been funded by the Grant-in-Aid for Challenging Exploratory Research of the Japan Society for the Promotion of Science (Grant No. 19654024). 


\section{References}

[Albert and Barabási 02] R. Albert and A.-L. Barabási. "Statistical Mechanics of Complex Networks." Rev. Mod. Phys. 74 (2002), 47-97.

[Boccaletti et al. 06] S. Boccaletti, V. Latora, Y. Moreno, M. Chavez, and D.-U. Hwang. "Complex Networks: Structure and Dynamics." Phys. Rep. 424 (2006), 175308.

[Boguñá and Pastor-Satorras 03] M. Boguñá, and R. Pastor-Satorras. "Class of Correlated Random Networks with Hidden Variables." Phys. Rev. E 68 (2003), 036112.

[Bose and Sen 07] A. Bose and A. Sen. "On Asymptotic Properties of the Rank of a Special Random Adjacency Matrix." Elect. Comm. in Probab. 12 (2007), 200-205.

[Caldarelli et al. 02] G. Caldarelli, A. Capocci, P. De Los Rios, and M. A. Muñoz. "Scale-Free Networks from Varying Vertex Intrinsic Fitness." Phys. Rev. Lett. 89 (2002), 258702.

[Diaconis et al. 09] P. Diaconis, S. Holmes, and S. Janson. "Threshold Graph Limits and Random Threshold Graphs." Internet Mathematics 5:3 (2009), 267-318.

[Fujihara et al. 09a] A. Fujihara, Y. Ide, N. Konno, N. Masuda, H. Miwa, and M. Uchida. "Limit Theorems for the Average Distance and the Degree Distribution of the Threshold Network Model." Interdisciplinary Information Sciences 15:3 (2009), 361-366.

[Fujihara et al. 09b] A. Fujihara, M. Uchida, and H. Miwa. "Universal Power Laws in Threshold Network Model: Theoretical Analysis Based on Extreme Value Theory." Physica A 389 (2009), 1124-1130.

[Hagberg et al. 06] A. Hagberg, D. A. Schult, and P. J. Swart. "Designing Threshold Networks with Given Structural and Dynamical Properties." Phys. Rev. E 74 (2006), 056116 .

[Hora and Obata 07] A. Hora and N. Obata. Quantum Probability and Spectral Analysis of Graphs. New York: Springer, 2007.

[Ide et al. 07] Y. Ide, N. Konno, and N. Masuda. "Limit Theorems for Some Statistics of a Generalized Threshold Network Model." Theory of Biomathematics and Its Applications III: RIMS Kokyuroku 1551 (2007), 81-86.

[Ide et al. 09] Y. Ide, N. Konno, and N. Masuda. "Statistical Properties of a Generalized Threshold Network Model." Methodol. Comput. Appl. Probab. 12:3 (2010), 361-377.

[Konno et al. 05] N. Konno, N. Masuda, R. Roy, and A. Sarkar. "Rigorous Results on the Threshold Network Model." J. Phys. A: Math. Gen. 38 (2005), 6277-6291.

[Mahadev and Peled 95] N. V. R. Mahadev, and U. N. Peled. Threshold Graphs and Related Topics. Amsterdam: Elsevier, 1995.

[Masuda and Konno 06] N. Masuda, and N. Konno. "VIP-Club Phenomenon: Emergence of Elites and Masterminds in Social Networks." Social Networks 28 (2006), 297-309. 
[Masuda et al. 04] N. Masuda, H. Miwa, and N. Konno. "Analysis of Scale-Free Networks Based on a Threshold Graph with Intrinsic Vertex Weights." Phys. Rev. E 70 (2004), 036124.

[Masuda et al. 05] N. Masuda, H. Miwa, and N. Konno. "Geographical Threshold Graphs with Small-World and Scale-Free Properties." Phys. Rev. E 71 (2005), 036108 .

[Merris 94] R. Merris. "Degree Maximal Graphs Are Laplacian Integral." Linear Algebr. Appl. 199 (1994), 381-389.

[Merris 98] R. Merris. "Laplacian Graph Eigenvectors." Linear Algebr. Appl. 278 (1998), 221-236.

[Newman 03] M. E. J. Newman. "The Structure and Function of Complex Networks." SIAM Rev. 45 (2003), 167-256.

[Servedio et al. 04] V. D. P. Servedio, G. Caldarelli, and P. Buttá. "Vertex Intrinsic Fitness: How to Produce Arbitrary Scale-Free Networks." Phys. Rev. E 70 (2004), 056126 .

[Söderberg 02] B. Söderberg. "General Formalism for Inhomogeneous Random Graphs." Phys. Rev. E 66 (2002), 066121.

[Taraskin 05] S. N. Taraskin. "Spectral Properties of Disordered Fully Connected Graphs." Phys. Rev. E 72 (2005), 056126.

Yusuke Ide, Faculty of Engineering, Kanagawa University, Yokohama, 221-8686, Japan (ide@kanagawa-u.ac.jp)

Norio Konno, Department of Applied Mathematics, Yokohama National University, Yokohama, 240-8501, Japan (konno@ynu.ac.jp)

Nobuaki Obata, Graduate School of Information Sciences, Tohoku University, Sendai, 980-8579, Japan (obata@math.is.tohoku.ac.jp)

Received December 31, 2009; accepted in revised form February 26, 2010. 